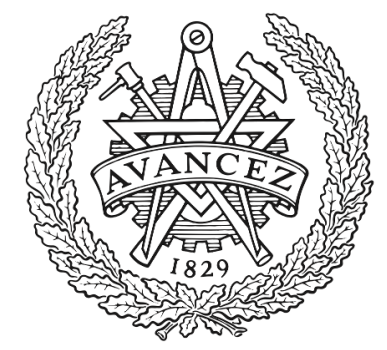

\title{
CHALMERS
}

UNIVERSITY OF TECHNOLOGY

\section{Digital Service Innovation from Open Data: Exploring the Value Proposition of an Open Data Marketplace}

Downloaded from: https://research.chalmers.se, 2023-04-26 09:54 UTC

Citation for the original published paper (version of record):

Smith, G., Ayaba Ofe, H., Sandberg, J. (2016). Digital Service Innovation from Open Data:

Exploring the Value Proposition of an Open Data

Marketplace. Proceedings of the 2016 49th Hawaii International Conference on System Sciences:

1277-1286. http://dx.doi.org/10.1109/HICSS.2016.162

N.B. When citing this work, cite the original published paper. 


\section{Digital Service Innovation from Open Data: Exploring the Value Proposition of an Open Data Marketplace}

\author{
Göran Smith \\ Viktoria Swedish ICT, Sweden \\ goran.smith@viktoria.se
}

\author{
Hosea Ayaba Ofe \\ Umeå University, Sweden \\ hosea.ofe@informatik.umu.se
}

\author{
Johan Sandberg \\ Umeå University, Sweden \\ jsberg@,informatik.umu.se
}

\begin{abstract}
Open data marketplaces have emerged as a mode of addressing open data adoption barriers. However, knowledge of how such marketplaces affect digital service innovation in open data ecosystems is limited. This paper explores their value proposition for open data users based on an exploratory case study. Five prominent perceived values are identified: lower task complexity, higher access to knowledge, increased possibilities to influence, lower risk and higher visibility. The impact on open data adoption barriers is analyzed and the consequences for ecosystem sustainability is discussed. The paper concludes that open data marketplaces can lower the threshold of using open data by providing better access to open data and associated support services, and by increasing knowledge transfer within the ecosystem.
\end{abstract}

\section{Introduction}

Open data is often described as an enabler of economic growth due to its high potential for digital service innovation $[1,2]$. In a recent report, it was for example estimated that open data could generate more than $\$ 3$ trillion world-wide in additional value across seven application domains [3]. Beyond financial gains, digital service innovation from open data can also have positive societal effects by increasing government transparency [4, 5], quantity and quality of public services [6] and interaction between stakeholders [7] as well as empowering both data users and providers [8].

Contemporary organizations generate vast amount of data in their daily activities [9-12]. With the growing expectation of value generation from this data, the public sector is increasingly publishing datasets at both local and national levels [13]. Contributing factors to this trend are the growing public demand as well as initiatives pushing public organizations to publish data such as the directive on the re-use of public sector information in the EU and the open government initiative in the US.
Much of the high expectations on value generation from open data are however yet to be realized [14]. Both data providers and users are facing adoption barriers, hindering sustainable ecosystems to emerge. Examples of such barriers include institutional aspects, task complexity, use and participation, legislation, information quality and technical details $[15,16]$.

With the growing realization that service innovation based on open data requires more than mere provision of data [15], novel measures for facilitating data use are developing. An intermediary form of organizing interactions that has drawn attention recently is the open data marketplace [7, 17]. Intermediary platforms constitute marketplaces by providing requisite infrastructure, rules and services for transactions of data, knowledge and experiences between open data providers and users [18].

While extant research provides an understanding of why increased interactions might stimulate digital service innovation, little is known of how marketplaces affects interaction patterns [7, 19]. Furthermore, the little research that has been done largely neglects the data user perspective. Accordingly, this paper examines the following research question: how does open data marketplaces generate value for open data users?

This issue is explored through a study of an open data marketplace in the public transport industry. To understand the value proposition of the marketplace, its sociotechnical structures and practices are first examined before the values open data users perceive from it are analyzed.

In the following, a brief overview of extant research on open data ecosystems and open data adoption barriers is first provided. Research setting and method are then presented prior to the analysis of the elements of the studied marketplace and the open data users' perceived values. Finally the paper discusses how the identified elements and values impact previously identified adoption barriers and what implications open data marketplaces can have on the sustainability of open data ecosystems. 


\section{Theoretical framework}

\subsection{The open data ecosystem}

There are many different definitions and understandings of open data as it is still a nascent and emerging phenomenon. While some argue that the open data label requires public entities to comply with strict principles in terms of e.g. granularity and accessibility [20], others suggest more operational definitions based on the release of data across organizational boundaries $[4,14,21]$. For the purpose of this study, the definition of open data as "nonprivacy-restricted and non-confidential data that is produced with public money and is made available without any restrictions on its usage and distribution" is adopted [15, p. 238].

Open data production, distribution and consumption has been suggested to take place in a six step process: (1) creation of data, (2) publication of data, (3) finding data, (4) analysis of data, (5) data processing and (6) feedback and discussions on open data [16]. As illustrated in figure 1, data providers manage step 1-2 and data users manage step 3-5, while the sixth is a joint activity.

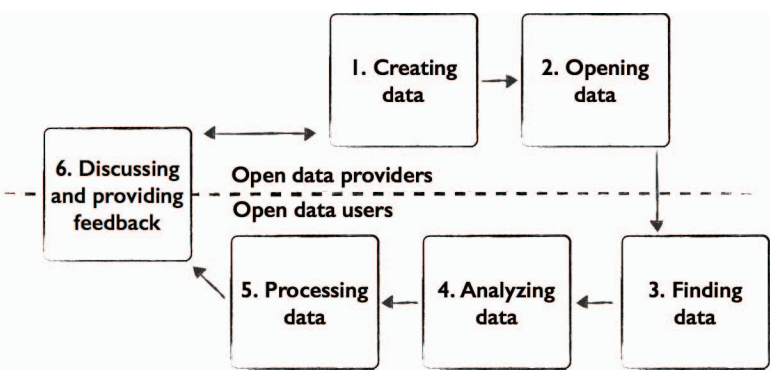

\section{Figure 1: The open data process [Adopted from 5]}

For digital service innovation to take place, open data providers rely on distributed data users, hoping to reap advantages at the intersection of specializations [7, 21-24]. Innovation processes in general and digital innovation in particular is increasingly relying on distributed organizing logics that doesn't conform to organizational boundaries [25-27]. Instead, digital innovation is often doubly distributed in the sense that control over technological resources is spread across multiple entities, and that knowledge is dispersed across heterogeneous disciplines and communities [28].

These loosely coupled networks of actors are often referred to as ecosystems. In open data ecosystems, the key stakeholders are open data providers (supplying raw or linked data), open data users (direct data users or service developers) and open data service end users [23]. Additionally, intermediaries such as, open data consultants, open data brokers and open data marketplaces can play important roles in the ecosystem by influencing all of the steps in the open data process. Open data consultants advise stakeholders on options and possibilities [23] while open data brokers arrange links between open data providers and users [21]. Both consultants and brokers can smoothen interactions by providing services for specific and well-formulated problems. Thus, they mainly facilitate exploitative innovation [29, 30]. Open data marketplaces instead provide structures that are more permanent in time and spurs enduring explorative innovation efforts by generating more and better options for trading data [30-32]. The relationships between actors in the open data ecosystem are illustrated in figure 2 .

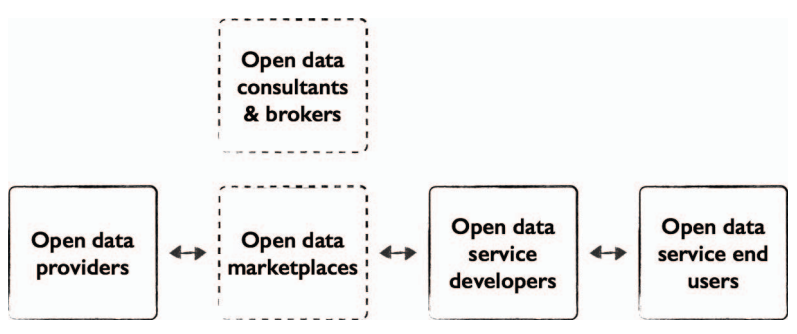

Figure 2: The open data ecosystem

\subsection{The role of open data marketplaces}

The purpose of an open data marketplace is to facilitate "trading and sharing of open data and data services including advice and assistance in an open cooperative environment" [7, p. 310]. Digital marketplaces in general provide technological infrastructure, rules, business models and services for transactions between providers and consumers [18]. To this end, an open data marketplace encompasses a technical platform with the capacity to link data providers and users. A marketplace also contains policies for conducting transactions, a business model and services such as data transformation and/or support functions [7].

Since open data marketplaces are two-sided in that they link providers and users, they are subject to network effects. That is, the value of the system is affected by other actors' participation or nonparticipation [7, 33]. Based on research on platforms and two-sided markets, mainly positive network effects are expected, both on the same side as well as crossside [34]. That is, for both data providers and users the value of the marketplace generally increases by more actors on either side of the market. 
Open data marketplaces enable transactions by providing social and technical structures that can be reused to lower costs involved with barriers for exchanging data, knowledge and experience [7]. These costs are mainly non-monetary and instead involves time, access and learning [24, 35]. The underlying premise behind the formation of open data marketplaces is that by addressing barriers for trade, interactions will increase and the ecosystem will become more sustainable.

\subsection{Open data adoption barriers}

Research on impediments for open data ecosystems to develop and thrive has identified six types of salient barriers; institutional aspects, task complexity, use and participation, legislation, information quality and technical details [15]. These barriers can each affect one or multiple steps in the open data process. While some of them are likely to be addressed by one or multiple of the market elements, others such as legislation are likely to be geographically dependent, domain specific and technically administered by data providers. In the following, each of these categories of barriers is discussed in more detail.

Institutional barriers refer to organizational unwillingness to change, often stemming from a high focus on accountability as compared to entrepreneurial activities [36]. The institutional barriers are located within the data providers and can result in unwillingness to publish data, insufficient resources for development of data and lack of ability to respond to user input [15]. Institutional barriers can severely impact data users by resulting in insufficient, poorly structured or low quality data.

Task complexity barriers refer to challenges involved with finding, analyzing and processing published data [15]. Data users face these barriers but they also affect providers by limiting the effectiveness of service innovation. They stem from design decisions on how the data is captured, stored and published and are often due to lack of standards and understanding of potential use scenarios.

Use and participation barriers refer to ease and attractiveness of joining and contributing to the open data ecosystem. Underlying factors raising barriers in this area include lack of incentives, lack of ability or time to engage in an ecosystem, costs and competition from other ecosystems [15]. Data users might be motivated to participate in a specific ecosystem by monetary reasons but also by non-monetary such as learning, problem solving, and enjoyment [37-39].

Legislation barriers concern privacy issues, disputes and litigations, security, licenses and reuse of contracts and agreements [15]. These barriers involve both legal and technical issues, while the first normally concern formal agreements that can be managed through standardized licenses, the latter is inherently dynamic. For example, as technological capability increases, data that once was considered fully anonymised can become personal data as new functionality allows identification [40].

Information quality related barriers arise from poorly captured, maintained and described data, or from too high demands on data users' data processing skills. $[11,15]$ When the data does not meet sufficient levels of detail due to e.g. being incomplete, obsolete or poorly structured, efforts to develop services become fruitless.

Finally, technical details can pose challenge for example through dispersion of data across multiple entities due to lack of a central portal, absence of standards, fragmentation of applications or lack of meta standards [15].

\section{Research approach}

\subsection{Research setting}

Trafiklab is an open data marketplace distributing open public transport data, linking together public transport authorities and open data users. The marketplace was developed in cooperation between Samtrafiken, Stockholm County Council and Viktoria Swedish ICT as a part of the 2010 - 2012 research program ISET (Innovation for sustainable everyday travel). It is currently under management of Samtrafiken and comprises eleven application programming interfaces (APIs) from three Swedish public transport authorities.

In addition to distributing available open public transport data, Trafiklab moreover intends to act as a community for open data users, as an initiative to catalyze further provision of open data from the public transport sector as well as a support function for transport authorities that want to disclose data on their own terms. The main goal of Trafiklab is to create value for public transport companies by facilitating development of digital travel services that are beneficiary for their customers. Samtrafiken vision is that Trafiklab eventually should become the leading venue for open public transportation data and related open data service development.

As of January 2015, Trafiklab had almost 3000 registered members. The median age was 36 years and more than nine out of ten members were men. The members had during the three-year lifetime of Trafiklab accumulated just above 2200 service development projects based on the distributed APIs. 


\subsection{Research method}

An explorative case study was deployed to provide insights on the value proposition of open data marketplaces. The empirical core of the study is narratives gathered in interviews with data users associated with Trafiklab. Additionally, the analysis is based on interviews with marketplaces personnel and data providers as well as on reviews of membership, website statistics, questionnaire answers, strategy documents and literature.

To gain a comprehensive understanding of Trafiklab's elements and strategies, the second and third author held nine semi-structured interviews with Trafiklab personnel during the spring of 2015. These interviews were complemented with secondary data such as internal strategy documents and six interviews with public transport organizations and persons involved in the founding and design of Trafiklab. Transcribed interviews and strategy documents were inductively coded following the recommendations in [41]. In total, this resulted in approximately 160 quotations and 40 codes. These were merged into four main recurring themes that reflected the key elements of Trafiklab. These elements are presented in section 4.1.

From the membership and website statistics that Samtrafiken gathers, it is possible to assess member characteristics, their activity on the website and their use of the APIs as well as aim and status of their registered service development projects. This knowledge was in this study used as a basis for understanding how the characteristics of questionnaire respondents and interviewed open data users differed from the average Trafiklab member.

Samtrafiken sent out a web-based questionnaire with 19 questions to the Trafiklab members (i.e. open data users) in the fall of 2014. The main aim of the questionnaire was to get open data users' input on what to prioritize in the future development of Trafiklab. The questionnaire contained three open-ended questions and 16 multiple-choice questions. It generated responses from 84 open data users.

During spring 2015 the first author conducted 19 semi-structured interviews with questionnaire respondents that had signaled their willingness to participate in further assessments. The interviews followed an interview guide with five overarching discussion topics: interviewee background information, motivations for developing services and for using Trafiklab, service development process, experiences of using Trafiklab and Trafiklab's areas of improvement.

Following the process in [41], the analysis of the collected data resulted in approximately 1400 quotations, 350 codes and 70 themes. Five of the most prominent themes that concerned the value for open data users are presented in section 4.2.

Lastly, the identified elements and perceived values were compared with existing literature on open data adoption barriers. The categorization of open data adoption barriers in [15] was used as framework for evaluating whether different elements of open data marketplaces address recognized problem areas and in that case have potential to facilitate open data use.

\section{Results}

\subsection{Elements of the marketplace}

Numerous aspects of Trafiklab's activities and strategies were identified during interviews with the Trafiklab personnel. In this section, four key elements are described briefly. An overview of the elements is presented in Table 1 .

\section{Table 1. Elements of the marketplace}

\begin{tabular}{|c|c|}
\hline Element & Content \\
\hline $\begin{array}{l}\text { Technical } \\
\text { platform }\end{array}$ & $\begin{array}{l}\text { The technical platform encompasses a back- } \\
\text { end of the website and an API management } \\
\text { system that handles access to APIs and } \\
\text { gathers statistics. }\end{array}$ \\
\hline Website & $\begin{array}{l}\text { The website includes API descriptions and } \\
\text { documentations, operational statuses and } \\
\text { access to support services as well as a news } \\
\text { section, project and member catalogues and } \\
\text { links to other API-sources. }\end{array}$ \\
\hline $\begin{array}{l}\text { Support } \\
\text { services }\end{array}$ & $\begin{array}{l}\text { The support services consist of } \\
\text { documentation and code examples, a support } \\
\text { forum with tier one support, operational } \\
\text { statuses of the APIs, gathering of usage } \\
\text { statistics and user requests, data quality } \\
\text { check and information on upcoming updates. }\end{array}$ \\
\hline $\begin{array}{l}\text { Knowledge } \\
\text { sharing } \\
\text { activities }\end{array}$ & $\begin{array}{l}\text { The marketplace showcases projects and } \\
\text { members, communicates open data news } \\
\text { through newsletters, blogs and social media, } \\
\text { arranges meet-ups and partners with digital } \\
\text { innovation contests. }\end{array}$ \\
\hline
\end{tabular}

4.1.1. Technical platform. Trafiklab offer data providers two alternative solutions for publishing their APIs on the marketplace. Trafiklab can either manage the API through its API management system or just present the API at the website (see figure 3). When the first option is picked, Trafiklab manages the access to APIs and the amount of calls by assigning project specific API keys. Some of the APIs have assigned maximum allowed amounts of requests per project and month. This number can be enlarged if the open data user applies for an API key upgrade, which in turn can only be done upon verification that the service have a 
certain amount of end-users. Thus, the API management system is used by Trafiklab both as a tool for providing access to APIs and to identify which data users and projects that utilizes the APIs, and also as a proxy instrument to protect the data providers' backend systems from being overloaded with unnecessary requests. Trafiklab furthermore uses the API management system to monitor and analyze API performance and errors.

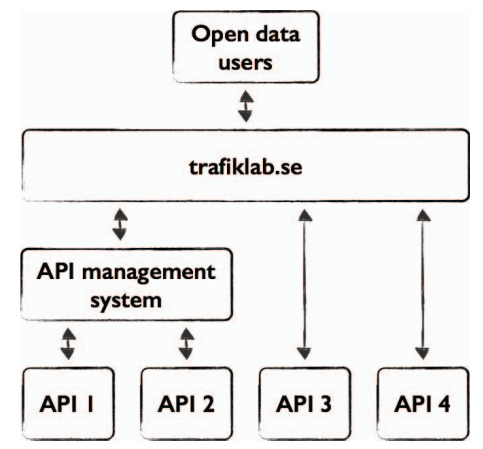

Figure 3: Structure

4.1.2. Website. Registered members can obtain access to Trafiklab's APIs through the website. This requires first registering development projects and then applying for API keys for these projects. In addition, the website contains fundamental resources for retrieving and understanding the APIs. These resources include descriptions, documentations and code examples for the APIs, their operational statuses and a link to an external support forum. Members as well as all ongoing and completed development projects are furthermore exhibited on the website. Lastly, the website provides links to other API sources and it is possible to read about upcoming activities and the like in the newsfeed. Hence, the website is supposed to serve as a comprehensive hub for service development based on open public transport data.

4.1.3. Support services. Trafiklab supports public transport organizations that want to disclose their data by offering open data competence and a technical platform for distributing the data. Trafiklab also provide a conducive environment for data providers for testing their data quality prior to release. This is made through automatic test scripts. Thus, Trafiklab hopes that data providers without appropriate human or technical resources can release their datasets. Furthermore, Trafiklab gives associated data providers feedback on API usage, up-time statistics as well as on complaints and requests from open data users.

Trafiklab provides tier one support to open data users. The main channel for this is an external support forum that can be accessed through the website. Through this support forum, data users can ask questions or propose and discuss suggestions for improvement. Tier one support is further enhanced through direct contact with Trafiklab's personnel at meet-ups and through e-mail communication. In cases where Trafiklab is unable to answer questions, it uses its close interaction with data providers to identify whom those questions could be forwarded to. In so doing, Trafiklab acts as both a direct support channel for data users as well as an agent blurring the boundaries between data users and providers in the open data ecosystem. This is supposed to reduce the staffing needs of the data providers and increase access to appropriate support for data users.

Descriptions, documentations, code examples and operational statuses for the APIs are displayed on the website to increase the understanding of the APIs. In order to keep data users aware of ongoing development in the open data ecosystem, Trafiklab also communicates upcoming changes on the technical platform and the APIs through newsletters, blogs and social media.

4.1.4. Knowledge sharing activities. The newsletters, the blog and the meet-ups are not only used to communicate information on upcoming changes, but also to inform on the open data scene in general and to showcase examples and opportunities. The main reasons for doing that are to inspire and motivate.

The meet-ups, which are usually arranged every third month, are further aimed at stimulating interaction between stakeholders and to increase their involvement and insight in the development of the marketplace. At these meet-ups, data users interact face-to-face with other data users, exchange ideas on applications and share their motivation behind those applications. Furthermore, arranging meet-ups is also a means of gathering users' input and attracting new members to the community.

Showcasing members and service development on the website is meant to display the results of the marketplace and the data, both for the data providers and users as well as for the general as a whole.

Lastly, Trafiklab organizes innovation contests together with other actors in the public transport industry. During such contests, data users jointly develop services over a limited period of time. Besides using the contests to inform the broader public of Trafiklab's activities, they are also intended to, by the means of inspiration and motivation, increase the diversity of the community around Trafiklab and of services developed. 


\subsection{Perceived values}

A vast number of perceived values, resulting from introducing Trafiklab into the open data ecosystem, were expressed in the questionnaire responses and in interviews with open data users. Five of the most prominent perceived values are presented below. An overview of them is shown in table 2.

\section{Table 2: Perceived values}

\begin{tabular}{|c|c|}
\hline Perceived value & Description \\
\hline $\begin{array}{l}\text { Lower task } \\
\text { complexity }\end{array}$ & $\begin{array}{l}\text { Trafiklab lowers the threshold for } \\
\text { finding, understanding and using } \\
\text { open public transport data as well } \\
\text { as for getting access to appropriate } \\
\text { support functions. }\end{array}$ \\
\hline $\begin{array}{l}\text { Higher access to } \\
\text { knowledge }\end{array}$ & $\begin{array}{l}\text { Trafiklab increases open data users' } \\
\text { access to knowledge from open } \\
\text { data providers and open data } \\
\text { expertise as well as from other } \\
\text { open data users and their } \\
\text { development projects. }\end{array}$ \\
\hline $\begin{array}{l}\text { Increased } \\
\text { possibilities to } \\
\text { influence }\end{array}$ & $\begin{array}{l}\text { Trafiklab provides an augmented } \\
\text { channel for communicating needs } \\
\text { to data providers and for } \\
\text { influencing the open data } \\
\text { provision. }\end{array}$ \\
\hline Lower risk & $\begin{array}{l}\text { Trafiklab lowers the perceived risk } \\
\text { involved with building services } \\
\text { based on open public transport } \\
\text { data. }\end{array}$ \\
\hline Higher visibility & $\begin{array}{l}\text { Trafiklab demonstrates that the } \\
\text { data providers believe in open } \\
\text { innovation and showcases the } \\
\text { positive effects of disclosing data. }\end{array}$ \\
\hline
\end{tabular}

4.2.1. Lower task complexity. One of the major differences that open data users have witnessed with the introduction of Trafiklab is the reduced number of data provision websites and actors to interact with. Prior to Trafiklab's emergence as a market for open data, data for various distributed public transport authorities' was found on their websites, Trafiklab offers data users a central portal for accessing Swedish open public transport data. This simple but distinct difference makes it easier for open data users to find and gain access to open data.

"The basis is that we want access to the data and Trafiklab is thus a great time saver. It would have been demanding to have to reach out to all public transport companies in the country." - Data user 4

The compilation and structured presentation of open data at Trafiklab also makes it less cumbersome for open data users to browse and compare different
APIs. Hence, it is now easier for them to get an overview of available open public transport data and to identify the most appropriate APIs for their purposes.

"It is a great place to compare the different APIS and to see which one fits best." - Data user 6

Understanding and making use of the data as well as maintaining developed services have also been facilitated with Trafiklab's emergence. This is due to that Trafiklab additionally to compiling and presenting open data also offers documentation, operating status and tier one support for the APIs. Open data users can therefore find more of the fundamental resources for service development at one spot.

4.2.2. Higher access to knowledge. Trafiklab does not only compile open data and support services, but also brings together open data providers and open data users in knowledge sharing activities. One of the consequences is that open data users gain better exposure and access to the knowledge of others. This knowledge is partially transferred at meet-ups, at the support forum and during individual interactions, but also when open data users are studying other Trafiklab related development projects. The perceived effects among open data users are inspiration, motivation and guidance for pursuing their project ideas.

"They talked about this journey planner made for those who have been diagnosed with Aspberger when I was there - Resledaren. I think it is absolutely superb. It also provides inspiration." - Data user 5

Furthermore, Trafiklab communicate news regarding open data through meet-ups, newsletters and social media. The open data users thus experience a level of certainty in the open data ecosystem as Trafiklab gives them a higher awareness of current possibilities and of what is to be expected in the future. This enhances their opportunities to identify appropriate project ideas. It also makes their development processes more efficient and stable since it has become easier to plan them.

"An important part is also to be able to see where all this is going, how safe it is, how changeable it is and what is going to come in the future." - Data user 3

4.2.3. Increased possibilities to influence. Unlike the open data providers, Trafiklab is an intermediary organization focused on facilitating innovation based on open public transport data. Trafiklab has therefore acquired a role in the ecosystem where it does not only act as a neutral marketplace but also as a proponent, 
advocating the interests of open data users. Trafiklab gathers their issues and requests and conveys them to data providers. This is applauded by open data users since it is ought to lead to more open data and higher data quality. The common understanding is also that Trafiklab can emphasis the importance of key issues in a way that a single user cannot.

"They also collect requests in a way that makes it possible to take them forward with a bit more weight." - Data user 3

However, Trafiklab does also aid single users who want to communicate their needs directly. Interviewees expressed that is now easier to identify appropriate people at the data providing organizations since they are involved with Trafiklab, and to meet them face-toface since Trafiklab arranges meet-ups.

"It is a means for establishing contacts, which is pretty neat when dealing with organizations such as the Swedish Transport Administration. It can be very difficult to find the right person otherwise." - Data user 3

4.2.4. Lower risk. As an intermediary actor in the open data ecosystem, Trafiklab dampens negative impacts when data providers alter their APIs. This is done partly through Trafiklab's API management system, which takes care of API calls and forwards them to the various data providers' backend systems, but mainly through Trafiklab's frequent contact with open data providers and fast and clear communication on upcoming changes.

"They have a good structure and clearly announce when changes are about to come. I feel that they have frequent contact with users and ask or remind that: now will this change happen - be sure to make the changes needed for it to continue to function." - Data user 18

A consequence of the community aspect of Trafiklab is that it has become visible for the open data users that other people are using the APIs too. Hence, they experience a sense of safety in numbers regarding the long-term stability of the data provision service. I.e. that the large pool of users would make it troublesome for the open data providers to shut down or implement significant changes of their APIs without prior notice.

"The main value is the data. Maybe to some extent also the packaging - that it is available in a certain way, that you can trust it and that there are more people using it the same way, creating a sense of safety regarding that I will be able to download it in this way and that API will live on." - Data user 11

4.2.5. Higher visibility. Many of the data users consider open government data as a valuable part of the democratic process. Trafiklab is one initiative in that direction. More than that, the existence of Trafiklab is also a symbolic move acknowledging that the public transport companies together with customers can gain value from third-party developed services.

"I like the idea that they are so humble that they actually assume that there are other people out there who can do this, who can create better services than what they can internally."'- Data user 2

Trafiklab moreover showcases the effects of disclosing data, through the project catalogue and through the meet-ups during which successful projects often are presented. This feedback function is among the open data users believed to make it easier for data providers to motivate further open data initiatives.

"Trafiklab is also about ensuring that the Swedish Transport Administration, public transport authorities and operators understand this - understand the importance of open data, what open data services that are developed and what value they actually have." Data user 3

\section{Discussion}

\subsection{Impact on open data adoption barriers}

This study sought to identify perceived values among the open data users from an open data marketplace and its constituent elements. To understand how open data marketplaces affect digital service innovation, the impact on previously identified adoption barriers for open data is discussed below. An overview of the impact is shown in table 3.

Table 3: Impact on open data adoption barriers

\begin{tabular}{ll}
\hline Adoption barrier & Impact \\
\hline Institutional & No impact \\
\hline Task complexity & Positive impact \\
\hline Use \& participation & Positive and negative impact \\
\hline Legislation & Positive impact \\
\hline Information quality & Positive and negative impact \\
\hline Technical & Positive impact \\
\hline
\end{tabular}


The introduction of Trafiklab has had a clear positive effect on the open data users' perception of task complexity barriers. The centralized portal for accessing data, support services and open data news makes it easier for open data users to discover, compare, understand, combine and make use of open public transport data. The higher access to the combined knowledge within the community also facilitates knowledge transfer, which result in an increased ability to overcome task complexity barriers such as understanding the meaning of data and combining datasets. A lower task complexity is thus generated both by the user-friendly and informative nature of the website as well as by support services and knowledge sharing activities.

By reducing the task complexity and by generating higher access to knowledge, Trafiklab has also reduced the impact of use and participation barriers such as lack of time and inadequate knowledge. Incentives and motivation to actively participate and to use the data have further increased due to the strengthened community, the higher ability to influence data providers, the higher visibility and the lower perceived risk. However, an unintended negative effect of that the data have to pass Trafiklab's API management system in-between data providers and users is that some users do not perceive the data provision service as either scalable or long term sustainable. The amount of future requests is by these users believed to overload Trafiklab's technical platform, mainly since Trafiklab so far seems to advocate request-based APIs instead of enabling bulk download. The attractiveness of putting efforts into developing innovations based on the provided data is thus hampered for these users. Use and participation barriers are in other words influenced positively by the marketplace's website, support services and knowledge sharing activities but also negatively due to the additional intermediary platform.

Trafiklab has harmonized licenses and conditions for using the different APIs. Open data users complexity of obtaining comparable license for different datasets are lowered since it can be done using a repeatable procedure. Hence, in the perspective of open data users, legislation barriers are somewhat positively affected by the combined effect of the marketplace's technical platform and website.

Trafiklab has, as mentioned earlier, acquired a role where it does not solitary act as a neutral marketplace but also as a spokesman for the open data users' interests and as an agent blurring the boundaries between stakeholders. Hence it's easier for open data users to influence the data provision and open data providers gain better insight into obsolete, non-valid and missing data as well as other user requests. When interacting with data providers and when introducing new datasets to the marketplace, Trafiklab furthermore advocates harmonization of formats and procedures. Over time, these measures might lower information quality barriers as well as the technical barriers that relate to absence of standards.

However, some data users experience that the added intermediate step between data providers and data users, in conjunction with the request-based structure and format utilized by Trafiklab, in addition to risking the long-term sustainability also lowers data quality and access to raw data. Trafiklab's API management system seems to affect the uptimes and response times of the APIs negatively. It moreover enlarge the perceived distance between data users and the raw data since both Trafiklab's and the data providers' back-ends must be able to handle submitted requests in order for users to access data. Hence, the introduction of an additional technical platform might affect information quality barriers negatively, which in turn would lower the potential innovation height and distress task complexity barriers.

Open data users do moreover face several innovation barriers that are not listed in [15]. Particularly significant are for example barriers related to later stages in the service commercialization such as launching and marketing developed services. Trafiklab does not aid data users in these quests. However, it eases maintenance and adaptation of developed services by increasing the access to knowledge and support services.

\subsection{Consequences for ecosystem sustainability}

Based on the analysis of value generated by Trafiklab and the impact on adoption barriers, this paper suggests that open data marketplaces can have both transformative and performative effects on the ecosystem's sustainability [42, 43].

Much of the positive effects on the sustainability of the ecosystem generated from Trafiklab seems to refer to transformative innovation interactions. The knowledge sharing activities and support services spur knowledge creation and diffusion. It motivates use and participation by creating social structure for interaction and shared pieces of identity and by providing means to influence data providers. Trafiklab creates trust in the relationship between data providers and users by communicating changes and dampen levels of changes and their impact. These values to a high degree rely on Trafiklab's capacity to understand both the data provider and user perspective and provide mediating structures.

In terms of the performative aspects, the impact of Trafiklab is more mixed. Trafiklab does simplify data transactions by providing a central access point for 
data and support services. On the other hand, Trafiklab introduces yet another technical platform that the open data users depend upon and does not guarantee either uptime or long-term duration. Thus, it is difficult for open data users to guarantee a level of service to endusers, which impedes end-users willingness to pay. Trafiklab moreover does not aid open data users in marketing services to end-users, a major barrier for establishing sustainable business models according to interviewed data users. In terms of the performative aspects, Trafiklab do thus not address the data users' overarching economic sustainability problems, such as the difficulty of creating revenue and of being dependent on a data provision service without any requirements on its service quality.

\subsection{Implications}

Establishing an open data marketplaces can be an effective measure for facilitating innovation based on open data if adoption barriers such as task complexity or use and participation are impeding benefits to be reaped. It is however important to recognize that open data users face additional barriers which are not lowered by open data marketplaces. In order for a sustainable open data ecosystem to be established, open data users need help, not only with gaining access to the open data, but also with matters such as reaching out to customers.

It is moreover essential to appreciate that it is the combined effect of the elements of the marketplace that generate the proposed values for open data users. Data transactions are at the core of the service, but mere provision of data does not generate value on its own. Interviewed open data users expressed for example that the opportunities to meet other open data users face-to-face are highly valuable, suggesting that open data marketplace should establish physical meetings in addition to digital interactions. When setting up an open data marketplaces, it is also crucial to embed scalability in the technical platform and to ensure robustness in order to avoid hampering affects on service quality and a low long-term sustainability.

Limitations of the study include the multifaceted role Trafiklab has, making it uncertain whether the proposed values relate to specific functions of the marketplace or to their role as a proponent for user needs. Thus, in order to verify the findings and provide deeper understanding of the value propositions, marketplaces in other open data ecosystems should be examined. Furthermore, the value propositions for data providers' and other stakeholders must be analyzed in order to understand the full effect of marketplaces on the open data ecosystem.

\section{Conclusion}

The main value proposition of an open data marketplace for open data users is that the central portal provides better access to open data and associated support services. This value is enabled by the technical platform, the website and the support services. However, an open data marketplace can additionally also bring together open data providers and open data users in the surrounding knowledge sharing activities. These activities increase the knowledge transfer within the ecosystem. The perceived values among open data users of this are: higher access to knowledge, lower perceived risk, higher ability to influence data provision and increased visibility of open data and its effects.

The generated values address several of previously identified open data adoption barriers, the most notably impacts being lower task complexity and increased attractiveness of using open data and actively participating in the ecosystem.

\section{References}

[1] M.T. Borzacchiello, and M. Craglia, "The impact on innovation of open access to spatial environmental information: A research strategy", International Journal of Technology Management 60.1, 2012, pp. 114-129.

[2] European Commission. "Communication from the Commission to the European Parliment, the council, the european economic and social committe and the committe of the regions - Open data: an engine for innovation growth and transparent governance", Communication 882, December, Brussels, Belgium, 2011.

[3] McKinsey, "Open data: Unlocking innovation and performance with liquid information", McKinsey, 2013.

[4] J.C. Bertot, P.T. Jaeger, and J.M. Grimes, "Using ICTs to create a culture of transparency: E-government and social media as openness and anti-corruption tools for societies", Government information quarterly 27.3, 2010, pp. 264-271.

[5] P. McDermott, "Building open government", Government Information Quarterly 27.4, 2010, pp. 401-413.

[6] Y. Charalabidis, , E. Ntanos, and F. Lampathaki, "An architectural framework for open governmental data for researchers and citizens". na, 2011.

[7] A. Zuiderwijk, E. Loukis, C. Alexopoulos, M. Janssen, and K. Jeffery, "Elements for the development of an open data marketplace", Conference for E-Democracy and Open Governement, 2014, pp. 309-322.

[8] A.C. Neuroni, R. Riedl, and J. Brugger, "Swiss Executive Authorities on Open Government Data - Policy Making beyond Transparency and Participation", IEEE, 46th Hawaii International Conference on Systems Sciences, 2013, pp. 1911-1920.

[9] T. Davies, "Open data, democracy and public sector reform." A look at open government data use from data. gov. uk, 2010. 
[10] K. Janssen, "The influence of the PSI directive on open government data: An overview of recent developments", Government Information Quarterly 28.4, 2011, pp. 446-456. [11] A. Zuiderwijk, M. Janssen, S. Choenni, R. Meijer, and R.S. Alibaks, "Socio-technical impediments of open data", Electronic Journal of eGovernment 10.2, 2012, pp. 156-172. [12] B. Ubaldi, "Open Government Data: Towards Empirical Analysis of Open Government Data Initiatives", OECD Working Papers on Public Governance, No. 22, OECD Publishing, Paris, France, 2012.

[13] P. Conradie, and S. Choenni, "Exploring process barriers to release public sector information in local government", Proceedings of the 6th International Conference on Theory and Practice of Electronic Governance, ACM, 2012, pp. 5-13.

[14] T. Davies, F. Perini, and J.M. Alonso, "Researching the Emerging Impact of Open Data. ODDC conceptual

framework", Open Data in Developing Countries, Working Paper 1, 2013.

[15] M. Janssen, Y. Charalabidis, A. Zuiderwijk, "Benefits, adoption barriers and myths of open data and open government", Information Systems Management 29.4, 2012, pp. 258-268.

[16] A. Zuiderwijk, and M. Janssen, "Barriers and development directions for the publication and usage of open data: A socio-technical view", Open Government, Springer New York, 2014, pp. 115-135.

[17] M. Janssen, and A. Zuiderwijk, "Infomediary business models for connecting open data providers and users", Social Science Computer Review 32.5, 2014, pp. 694-711.

[18] B.F. Schmid, and M.A. Lindemann, "Elements of a reference model for electronic markets", IEEE, Proceedings of the 35th Hawaii International Conference on Systems Sciences Vol. 4, 1998, pp. 193-201.

[19] J. Lindman, M. Rossi and K. V. Tuunainen "Open Data Services: Research Agenda", 46th Hawaii International Conference on System Sciences, 2013, pp. 1239-1246.

[20] Berners-Lee, T. (2006). Design issues: Linked data.

[21] A. Hjalmarsson, N. Johansson, and D. Rudmark, "Mind the Gap: Exploring Stakeholders' Value with Open Data Assessment", IEEE, 48th Hawaii International Conference on Systems Sciences, 2015, pp. 5-8.

[22] A. Latif, A, A.U. Saeed, P. Hoefler, A. Stocker, and C. Wagner, "The Linked Data Value Chain: A Lightweight Model for Business Engineers", I-SEMANTICS, 2009, pp. 568-575.

[23] Y. Tammisto, and J. Lindman, J, "Open Data Business Models", 34th Information Systems Seminar in Scandinavia, Turku, Finland, 2011.

[24] Leonard-Barton, D., "Wellsprings of Knowledge: Building and Sustaining the Sources of Innovation", Harvard Business School Press, Boston, 1995.

[25] Chesbrough, H., W. Vanhaverbeke, and J. West, "Open innovation: Researching a new paradigm", Oxford University Press, Oxford, 2008.

[26] Y. Yoo, O. Henfridsson, and K. Lyytinen, "Research commentary: The new organizing logic of digital innovation: an agenda for information systems research", Information Systems Research 21.4, 2010, pp. 724-735.

[27] A. Bharadwaj, O.A. El Sawy, P.A. Pavlou, and N. Venkatraman, "Digital business strategy: toward a next generation of insights", Mis Quarterly, 37.2, 2013, pp. 471482.

[28] Y. Yoo, K. Lyytinen, and R. Boland, "Distributed innovation in classes of networks", IEEE, Hawaii International Conference on System Sciences, Proceedings of the 41st Annual, 2008, pp. 58-58.

[29] B.A. Gutek, A.D. Bhappu, M.A. Liao-Troth, and B. Cherry, "Distinguishing between service relationships and encounters", Journal of Applied Psychology, 84.2, 1999 pp. 218.

[30] J.G. March, "Exploration and exploitation in organizational learning", Organization Science 2, 1991. pp. 71-87.

[31] C.Y. Baldwin, and K.B. Clark, "The architecture of participation: Does code architecture mitigate free riding in the open source development model?", Management Science 52.7, 2006, pp. 1116-1127.

[32] J. Sandberg, L. Mathiassen, and N. Napier, "Digital Options Theory for IT Capability Investment", Journal of the Association for Information Systems 15.7, 2014, pp. 422453.

[33] Shapiro, C. and H.R. Varian, "Information Rules: A Strategic Guide to the Network Economy", Harvard Business Press, 1999.

[34] T., G. Eisenmann, G. Parker, and M.W. Van Alstyne, "Strategies for two-sided markets", Harvard Business Review 84.10, 2006, pp. 92.

[35] G. Magalhaes, C. Roseira, and S. Strover, "Open government data intermediaries: A terminology framework", ACM, Proceedings of the 7th International Conference on Theory and Practice of Electronic Governance, 2013, pp. 330-333.

[36] B. Bozeman, and G. Kingsley, "Risk culture in public and private organizations", Public Administration Review, 1998: pp. 109-118.

[37] M. Ceccagnoli, C, Forman, P. Huang, and D.J. Wu, "Co-creation of value in a platform ecosystem: The case of enterprise software", MIS Quarterly, Forthcoming, 2011.

[38] Y. Qiu, A. Gopal, and I.H. Hann, "Synthesizing Professional and Market Logics: A Study of Independent iOS App Entrepreneurs", ICIS 2011 Proceedings. Paper 10, 2011. [39] K.J. Boudreau, and L.B. Jeppesen, "Unpaid complementors and platform network effects? Evidence from on-line multi-player games", Social Science Research Network 16, April, 2011.

[40] A. Kulk, and B. Van Loenen, "Brave new open data world?", International Journal of Spatial Data Infrastructures Research 7, 2012, pp. 196-206.

[41] Charmaz, K., "Constructing Grounded Theory: A Practical Guide through Qualitative Analysis (introducing qualiatative method series)", Sage, London, 2006.

[42] J. Sandberg, J. Holmström, N. Napier, and P. Levén, "Balancing diversity in innovation networks: Trading zones in university-industry R\&D collaboration", European Journal of Innovation Management 18.1, 2015, pp. 44-69.

[43] K. Kellogg, W. Orlikowski, and J. Yates, "Life in the trading zone: Structuring coordination across boundaries in postbureaucratic organizations", Organization Science 17.1, 2006, pp. 22-44. 